\title{
Bayreuther Rainer, Engelberg Meinrad von, Rauschenbach Sina, Treskow Isabella von (dir.), Kritik in der Frühen Neuzeit. Intellektuelle avant la lettre
}

Wiesbaden : Harrassowitz Verlag, 2011, 404 p., $89 €$

\section{Christophe Losfeld}

\section{OpenEdition}

Journals

Édition électronique

URL : http://journals.openedition.org/ifha/7652

DOI : 10.4000/ifha.7652

ISSN : 2198-8943

Éditeur

IFRA - Institut franco-allemand (sciences historiques et sociales)

Référence électronique

Christophe Losfeld, «Bayreuther Rainer, Engelberg Meinrad von, Rauschenbach Sina, Treskow Isabella von (dir.), Kritik in der Frühen Neuzeit. Intellektuelle avant la lettre », Revue de l'IFHA [En ligne], Date de recension, mis en ligne le 15 décembre 2013, consulté le 22 septembre 2020. URL : http:// journals.openedition.org/ifha/7652; DOI : https://doi.org/10.4000/ifha.7652

Ce document a été généré automatiquement le 22 septembre 2020

(CIFHA 


\title{
Bayreuther Rainer, Engelberg Meinrad von, Rauschenbach Sina, Treskow Isabella von (dir.), Kritik in der Frühen Neuzeit. Intellektuelle avant la lettre
}

Wiesbaden : Harrassowitz Verlag, 2011, 404 p., $89 €$

\author{
Christophe Losfeld
}

Il n'est pas ici le lieu de discuter si l'intérêt croissant porté au phénomène des intellectuels tient à leur perte de prestige ou d'influence dans les sociétés contemporaines. Mais force est de constater qu'ils ont fait, depuis quelques années, l'objet d'études qui en ont permis une compréhension plus approfondie, et qui relativisent, ou du moins modifient, l'image qu'on s'en était forgée. Initialement, en effet, le terme d'intellectuel est péjoratif, qu'appliquaient les antidreyfusards à leurs adversaires, cette gente prétendument peu rouée aux mécanismes du politique et qui prétendait intervenir dans des questions justement politiques ou encore sociales en s'appuyant sur le poids de l'opinion publique. Repris par les auteurs que ce terme devait disqualifier, la notion d'intellectuel a servi à désigner ceux qui, quittant leur domaine d'activité originel, soumettaient à la critique une réalité perçue comme déficiente et qu'ils œuvraient, justement, à changer. Comme le rappellent avec raison Rainer Bayreuther, Meinrad von Engelberg, Sina Rauschenbach et Isabella von Treskow dans l'excellente introduction de Kritik in der frühen Neuzeit. Intellektuelle avant la lettre, la perception du phénomène des intellectuels varie selon les contextes nationaux : alors que la recherche en France, par exemple, reste généralement fidèle à l'interprétation première de ce qu'est un intellectuel, insistant sur la critique formulée, au nom de la " justice ", à l'encontre des pouvoirs en place, les Allemands intègrent davantage à leur conception de l'intellectuel le poids du savoir et de sa transmission. A la faveur d'une telle évolution, le terme d'« intellectuel » a commencé à être employé pour désigner 
l'attitude d'écrivains ou de penseurs antérieurs à la naissance proprement dite des intellectuels et à être utilisé pour le XIXe, le XVIIIe, voire pour le Moyen Âge et ce au prix d'une certaine imprécision du terme.

Ce serait faire injure à Kritik in der frühen Neuzeit que d'y voir simplement une tentative nouvelle de mettre en lumière des formes d'engagement intellectuel avant que ce dernier n'ait existé stricto sensu. Ce serait faire injure aussi à ses éditeurs, à leur rigueur scientifique et leur conscience méthodique. Parfaitement conscients du caractère spécifique de l'époque qui vit naître les intellectuels, ils se sont, bien davantage, proposé d'examiner premièrement s'il est propre aux Lumières ou à l'époque moderne de voir des penseurs intervenir dans des questions touchant à la politique ou au social (ce que les éditeurs appellent « eingreifendes Denken ») ou si un tel phénomène peut être constaté dans le contexte d'autres structures sociales où, en particulier, dans celles où la notion d'opinion publique n'avait pas l'importance qu'on lui a attribuée depuis lors. En second lieu, ils se sont interrogés pour déterminer par quels médiums une démarche intellectuelle est possible.

Les douze contributions rassemblées dans cet ouvrage ont tenté d'apporter une réponse à ce double questionnement en prenant en considération des sujets concernant l'ancien comme le nouveau monde, des sujets couvrant une vaste période menant $\mathrm{du}$ XIVe au XVIIIe, et dépassant, enfin, largement les textes imprimés. C'est ainsi que $\mathrm{E}$. Bayreuther a montré combien la musique, dès le Moyen-Âge peut participer d'une critique de la politique dont elle dénonce les insuffisances au nom des droits de l'individu («Strukturen politischer Kritik in der Musik des späten Mittelalters und der Frühen Neuzeit »).

Dieter Jansen présente à propos de la guerre les thèses de Francisco de Vitoria, d'une part, et celles d'Erasme, de l'autre, et donc deux argumentations qui illustrent parfaitement le passage de la position de l'érudition classique à une forme d'intellectualisme ( «Intellektuelle und die Auseinandersetzung mit dem Krieg in der Frühen Neuzeit »).

Se fondant sur l'exemple des débats menés tant sur les Indiens au XVIe siècle que, un siècle plus tard sur le retour des Juifs en Angleterre (deux sujets apparemment hétéroclites, mais, en définitive interdépendants à cause du rôle joué, dans les discussions sur les Indiens, par le rabbin Menasse ben Israel) Sina Rauschenbachen présente également un tel glissement : à la faveur de ces débats respectifs, on constate que certains érudits commencent à s'adresser à un public plus vaste, quittant, par conséquent, l'arène traditionnelle des controverses érudites, même s'ils restent marqués par les catégories de pensée de leur époque, comme cela apparaît, par exemple, dans la fine analyse que propose S. Rauschenbach de la conscience (" Von der gelehrten zur intellektuellen Debatte. Die Indianer und die englische Diskussion über die Wiederzulassung der Juden im 17. Jahrhundert »).

Albert Schirrmeister, en prenant l'exemple d'Erasmus Stella, Johannes Cuspinian et Robert Gaguin, trois historiens humanistes, montre bien quelles sont les différences entre, d'une part, les humanistes soucieux de promouvoir un savoir utile à la Cour et dans les universités tout en participant de l'autonomie croissante de l'activité littéraire, et, d'autre part, les intellectuels prétendant, en vertu des capacités spécifiques dont ils disposent, pouvoir juger des réalités sociales et politiques. En un second temps, il détermine les conditions dans lesquelles les humanistes peuvent accéder au rang 
d'intellectuel («Wann können Humanisten Intellektuelle sein? Überlegungen anhand dreier Beispiele »).

Karsten Mackensen examine comment des musiciens œuvrant dans un contexte social différent de celui des intellectuels, ne fût-ce que parce que les premiers vivent encore dans un système marqué par le mécénat, peuvent cependant y échapper en se professionnalisant et en devenant théoriciens de la musique. Cela leur permet, en accédant au rang d'auteur voire d'érudit, de parvenir à une certaine autonomie, même si, pour cela, ils doivent accepter de dépendre du marché littéraire ( $"$ "... non servitio operis sed imperio speculationis..." Musiker in der Frühen Neuzeit zwischen Beruf und Bekenntnis »).

Ina Schabertrevient sur les conditions culturelles et religieuses qui ont permis de voir, dans l'Angleterre des XVIIe et XVIIIe siècles, l'émergence d'auteurs intervenant comme des intellectuels dans les débats de société (elle parle de "intellektuelle Einmischung »). Suite à la guerre civile, les structures traditionnelles se voient remises en question, obligeant les femmes à acquérir une certaine autonomie et à assumer des charges qui d'habitude revenaient aux hommes. Ce rôle grandissant des femmes est favorisé, enfin, par la réception du dualisme cartésien, qui permet, tout en continuant à affirmer la supériorité physique des hommes, d'insister sur l'égalité intellectuelle des deux sexes. Une telle évolution mène à la naissance d'intellectuelles, dont I. Schabert présente un tableau chatoyant ( « Die Frau als Intellektuelle im England des späten 17. und des 18. Jahrhunderts »).

Isabella von Treskow, elle, montre qu'étant données les structures sociales de l'Ancien Régime dans ce pays avant le dernier tiers du XVIIIe siècle, l'engagement intellectuel en France s'exprime surtout dans la littérature de fiction, et plus exactement dans les écarts aux normes qui peuvent être y être effectués. Et elle constate avec raison que l'étude des textes de fiction sous un tel angle ouvre de nouvelles perspectives de recherches poétologiques («Fiktion und intellektuelles Engagement. Zur Funktionsweisekritischer Intervention im Frankreich des 17. Jahrhunderts »).

Pour Ludwig Schwarte, l'engagement d'un intellectuel « avant la lettre » se caractérise par la volonté de créer un espace public (et différent, par conséquent, de l'espace communicationnel dans lequel il se meut sinon) au nom d'une vérité dont il nie qu'elle soit l'apanage du pouvoir. Bien au contraire, la critique intellectuelle peut être perçue avant tout comme la tentative de rompre, ou du moins de questionner, « le lien entre pouvoir et vérité » (p. 248). Il va de soi, dès lors, qu'un pareil engagement implique un intérêt profond pour les savoirs nouveaux comme pour les institutions qui en favorisent l'éploiement. Et L. Schwarte d'illustrer son raisonnement par une fine analyse des travaux sur de Nicolaus Steno sur l'anatomie cérébrale ( Von der Möglichkeit, die Wahrheit zu sagen. Intellektuelle, Experimentalwissenschaft und Öffentlichkeit um 1700 ...»).

Agir en intellectuel «avant la lettre» implique aussi que l'écrivain dispose d'une certaine autorité. En prenant l'exemple de J. J. Schleuzer, Ulrich Johannes Schneider montre comment cet auteur parvient à fonder doublement une telle autorité («Zur Rhetorik des Intellektuellen im 18. Jahrhundert. Johann Jakob Scheuchzer und seine Physica Sacra »).

Dorothea von Mücke, dans un article consacré à la généalogie du concept des intellectuels, s'intéresse à deux aspects fondamentaux de l'acceptation qu'on a d'eux : la critique formulée par une personne s'érigeant ou érigée en porte-parole, d'une part, 
et, de l'autre, le rôle central joué par un large public mature, prêt à participer aux débats et accordant sa confiance à un tel porte-parole, et ce, en dépit du fait que ce dernier ne saurait guère passer d'emblée pour spécialiste de la question qu'il défend ou qu'il n'est pas accréditée par le pouvoir en place. Un tel phénomène va de pair avec la transformation de la notion d'érudit, que D. v. Mücke décrit parfaitement en analysant le fameux texte de Kant sur les Lumières ainsi que deux autres de Lessing. À partir de la célèbre distinction entre " public » et " " privé », Kant forge une nouvelle définition de l'érudit qu'il fonde sur le lien que celui-ci entretient à un large public à la faveur d'une limitation de la censure et de la notion d'autorité (et D. v. Mücke évoque avec raison le rôle des prix d'académie dans cette évolution). Lessing, lui aussi, remet en question la compréhension traditionnelle de l'érudition au profit d'une « sécularisation de l'espace public »-«Säkularisierung der Öffentlichkeit » («Öffentlichkeiten der Aufklärung und intellektuelle Kritik »).

C'est une semblable modification de la notion d'érudit que retrace Hans-Jürgen Lüsebrink mais, cette fois, en prenant la France pour référence, même si l'on ne saurait parler d'une simple analogie, puisque, selon Lüsebrink, la France des Lumières représente « le contexte culturel le plus important » pour comprendre la naissance et le développement de l'intellectuel moderne, un intellectuel dont le précurseur est le "philosophe. En se penchant sur plusieurs figures exemplaires (en particulier Raynal et Madame du Châtelet), il peint le portrait de personnages anticonformistes, et qui font découler de cet anticonformisme des positions critiques annonciatrices de l'intellectuel («Vom "Gelehrten" zum philosophe. Selbstverständnis und Rollenbilder des "Intellektuellen" im französischen Aufklärungszeitalter »).

Partant du constat d'un lien étroit entre l'intellectuel, tel qu'il naît durant l'affaire Dreyfus, et la presse, Susanne Lachenicht s'interroge pour savoir quand un tel lien peut déjà être mis en lumière. Elle le met, finalement, en évidence dès la fin du XVIIIe siècle en étudiant les liens existant entre la presse, la Révolution et l'émergence de l'intellectuel. Elle constate, en effet, qu'à la faveur de la politisation de la société française durant les années qui ont précédé la Révolution, la presse est devenue le lieu d'expression de certains groupes, une évolution qui consacre la naissance du métier même de journaliste ( Periodische politische Presse und intellektuelle Intervention. Journalisten in Frankreich im späten 18. Jahrhundert »).

Meinrad von Engelberg, montre, lui, combien les peintres eux-mêmes peuvent intervenir dans les débats ou les questions qui préoccupent leur époque. De fait, comme la compréhension qu'ils ont de leur art permet une analogie avec la rhétorique (ce qu'Engelberg explique très bien en recourant au traité de Leon Battista Alberti), leurs productions peut devenir le lieu d'un message social et politique plus ou moins facile à décoder, comme le prouvent ses analyses d'œuvres de Piero della Francesca, Dürer, ou encore Goya ( ( Bildende Künste als Medien intellektueller Intervention »).

L'une des qualités de ce recueil consiste en ce que les éditeurs, dans une conclusion très éclairante, ont fait la synthèse de l'apport des différents articles et contribué, par là, à la question de savoir s'il est légitime de parler d'intellectuels avant la lettre. Six aspects résument à leurs yeux l'action de tels intellectuels au début de l'époque moderne : la critique (qui ne repose plus, comme au Moyen Âge, sur une compréhension normative de la vérité, mais fait, bien davantage place à l'individualité); cette critique peut s'exprimer par d'autres voies que par l'écrit, mais elle ne porte que lorsqu'elle se fonde sur la notion d'autorité. Elle s'accompagne aussi d'une modification de la signification 
du savoir (et donc de celle de l'érudit), dans la mesure où le critère d'utilité sociale gagne en importance) ainsi que d'un changement dans la compréhension de ce qu'est le public (car celui-ci change radicalement). Et, enfin, les éditeurs mettent bien en valeur que l'existence d'analogie avec la notion d'intellectuel telle que l'a forgée le XIXe siècle, il faut se garder de négliger les profondes différences entre l'intellectuel et "l'intellectuel avant la lettre ", ce que la question de l'autonomie suffirait à démontrer. Ces dernières réflexions prouvent bien la profonde rigueur scientifique du questionnement sous-tendant un recueil qui devrait constituer la source d'inspiration d'études futures et dont on ne peut que chaudement recommander la lecture.

INDEX

Thèmes : Kulturgeschichte

Index chronologique : Epochenübergreifend

\section{AUTEUR}

\section{CHRISTOPHE LOSFELD}

Martin-Luther Universität Halle-Wittenberg 